\title{
Monitoring of Persons with Risk for Exposure to Ebola Virus - United States, November 3, 2014-December 27, 2015
}

\author{
Hyacinte Julien Kabore, DDS ${ }^{1}$; Rodel Desamu-Thorpe, $\mathrm{MD}^{1}$; Lisa Jean-Charles, $\mathrm{MPH}^{1}$; Karrie-Ann Toews, MPH ${ }^{1}$; Rachel Nonkin Avchen, PhD ${ }^{1}$
}

During November 3, 2014-December 27, 2015, CDC implemented guidance on movement and monitoring of persons in the United States with potential exposure to Ebola virus (Ebola) (1). Monitoring was concluded in December 2015. After CDC modified the guidance for monitoring travelers from Guinea (the last country for which monitoring of travelers was recommended) in late December 2015, jurisdictional reports were no longer collected by CDC. This report documents the number of persons monitored as part of the effort to isolate, test, and, if necessary, treat symptomatic travelers and other persons in the United States who had risk for exposure to Ebola during the period the guidance was in effect. Sixty jurisdictions, including all 50 states, two local jurisdictions, and eight territories and freely associated states, reported a total of 29,789 persons monitored, with $>99 \%$ completing 21-day monitoring with no loss to follow-up exceeding 48 hours. No confirmed cases of imported Ebola were reported once monitoring was initiated. This landmark public health response demonstrates the robust infrastructure and sustained monitoring capacity of local, state, and territorial health authorities in the United States as a part of a response to an international public health emergency.

Monitoring of persons with risk for exposure to Ebola included active monitoring (daily reporting of temperature and other symptoms to public health officials) and direct active monitoring (daily reporting of temperature and other symptoms and daily direct observation by public health officials) (2). CDC defined three risk levels for the purpose of guiding monitoring and movement restrictions: "low but not zero risk" (low risk); "some risk," and "high risk." During November 3, 2014-March 9, 2015, reports to CDC consisted of individual-level daily submissions for all persons under monitoring from the included jurisdictions (2). After March 9, 2015, individual-level daily reporting was only submitted for symptomatic persons and persons with gaps in reporting exceeding 48 hours. Weekly aggregate monitoring data were collected from each jurisdiction for all persons under monitoring by epidemiologic risk category.

Complete monitoring (active monitoring or direct active monitoring) was defined as making contact with the monitored person, with no gaps of $>48$ hours in reporting of persons being actively monitored or in contact with persons receiving direct active monitoring (i.e., no loss to follow-up) during the 21-day monitoring period. The overall number of persons monitored included all persons who completed monitoring during the period of guidance implementation, in addition to any persons who left the United States before completing the full 21-day monitoring period and any persons under monitoring on December 27, 2015.

During November 3, 2014-December 27, 2015, in the 60 U.S. jurisdictions reporting, ${ }^{*} 29,789$ persons were monitored (Table). Overall, $97.0 \%$ of persons monitored were travelers at low risk, $1.5 \%$ were health care workers at low risk who provided patient care in the United States, and $1.6 \%$ were travelers at high or some risk (Figure 1). A median of 1,680 persons (range $=551-2,719$ ) were monitored in a given reporting week. Among health care workers at low risk, $61 \%$ were monitored during November-December 2014, and 36\% were monitored during March-April 2015, after caring for patients treated for Ebola in the United States. Among 442 persons at high or some risk (mostly health care workers who cared for patients in Ebola-affected countries), 90\% were monitored during November 2014-May 2015. The number of persons monitored weekly decreased $46 \%$ from a peak in mid-May 2015 to mid-June 2015. This decrease corresponded to the first declaration by the World Health Organization that Liberia was free of Ebola and CDC's subsequent modification of the monitoring recommendation to self-observation for travelers from Liberia. The number of persons monitored decreased a further 63\% during October-December 2015, after the United States stopped enhanced entry risk assessment and management for Liberia travelers and CDC's modification of monitoring guidance for Sierra Leone (Figure 1).

During a given week, a median of three persons for whom monitoring was indicated could not be contacted upon arriving in the jurisdiction responsible for their monitoring $(0.3 \%$; range $=0-48$ persons per week). Among all persons ever contacted for monitoring, a median of five persons had gaps in monitoring $>48$ hours in a given week $(0.3 \%$; range $=$ $0-26$ persons per week). The median number of persons with $>48$-hour gaps in monitoring declined over time and decreased from three persons per week (0.2\%) in February 2015 to two persons per week (0.1\%) in December 2015.

During a given week, a median of 11 persons who developed symptoms while under monitoring $(0.7 \%$,

\footnotetext{
* 50 U.S. states, District of Columbia, New York City, Puerto Rico, U.S. Virgin Islands, American Samoa, Guam, Federated States of Micronesia, Northern Mariana Islands, Palau, and Marshall Islands.
} 
TABLE. Ebola virus monitoring of persons with potential exposure, by epidemiologic risk category - 60 U.S. jurisdictions,* November 3, 2014December 27, 2015

\begin{tabular}{|c|c|c|c|c|}
\hline \multirow[b]{2}{*}{ Monitoring element } & \multirow[b]{2}{*}{ High risk/Some risk } & \multicolumn{2}{|c|}{ Low (but not zero) risk } & \multirow[b]{2}{*}{ Total } \\
\hline & & Travelers & U.S. HCWs & \\
\hline Type of daily monitoring & DAM & AM & DAM & - \\
\hline Reporting frequency to CDC & Daily/Weekly & Weekly & Weekly & - \\
\hline No. of persons monitored & 442 & 28,759 & 598 & $29,789^{\dagger}$ \\
\hline No. of jurisdictions conducting monitoring & 47 & 54 & 12 & $54^{\S}$ \\
\hline
\end{tabular}

Abbreviations: $\mathrm{AM}=$ active monitoring; $\mathrm{DAM}=$ direct active monitoring; $\mathrm{HCWs}=$ health care workers, including laboratory personnel.

* 50 U.S. states, District of Columbia, New York City, Puerto Rico, U.S. Virgin Islands, American Samoa, Guam, Federated States of Micronesia, Northern Mariana Islands, Palau, and Marshall Islands.

${ }^{\dagger}$ Adjusted for 10 persons whose risk category changed from some risk to low risk.

$\S \mathrm{A}$ jurisdiction could conduct monitoring of travelers in more than one risk category.

FIGURE 1. Number of persons $(\mathrm{N}=29,789)$ with potential exposure who were monitored for Ebola virus, by epidemiologic risk category and week - United States, November 3, 2014-December 27, 2015

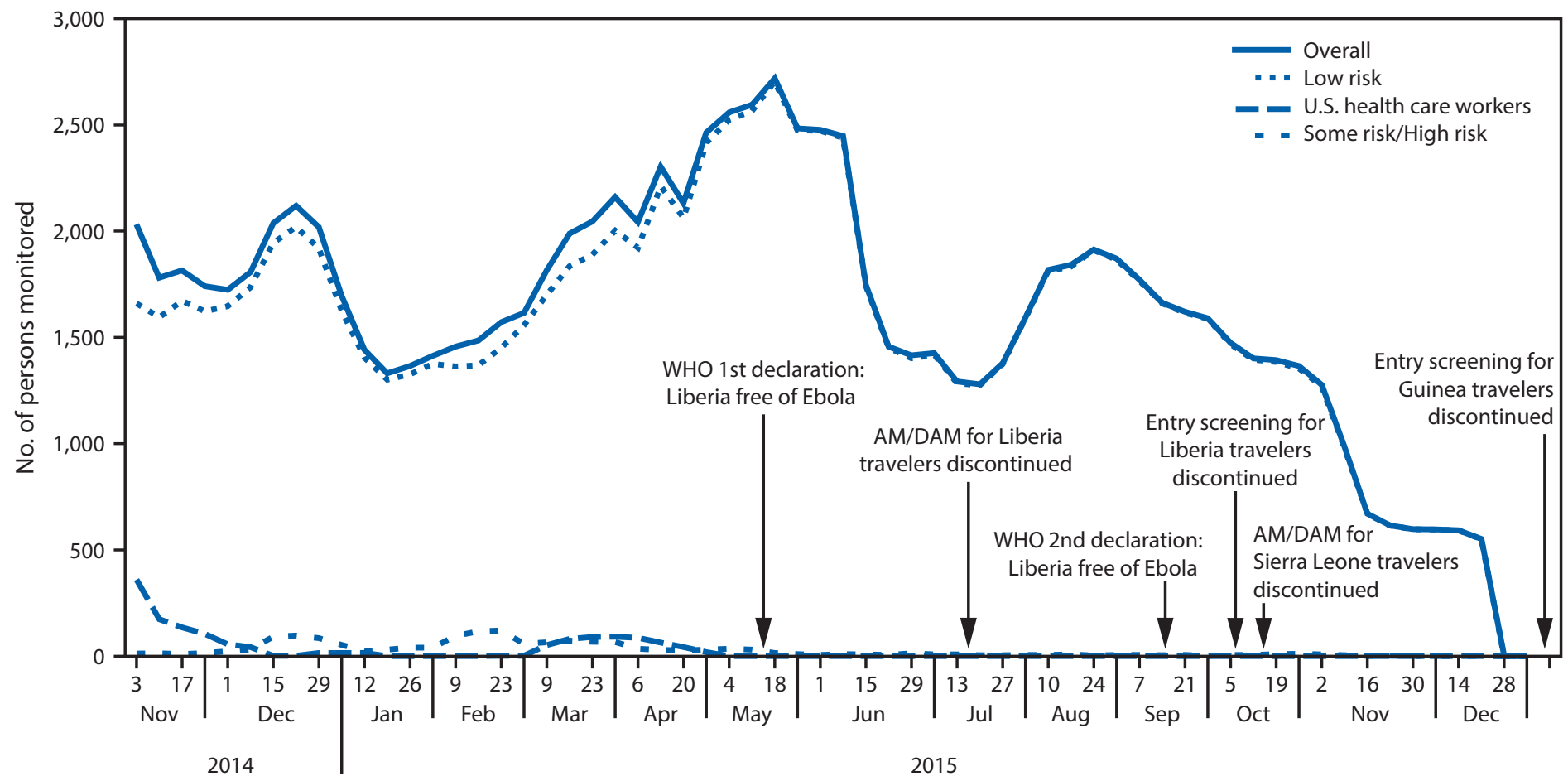

Week beginning/Year

Abbreviations: $\mathrm{AM} / \mathrm{DAM}=$ active monitoring/direct active monitoring; $\mathrm{WHO}=$ World Health Organization .

range $=1-43$ persons) were reported to CDC. Among 796 symptomatic persons in the low-risk and some-risk categories, $104(13 \%)$ were tested for Ebola during their monitoring period; none tested positive for Ebola. No persons at high risk reported Ebola-compatible symptoms.

All 50 states, plus the District of Columbia, New York City, Puerto Rico, and the U.S. Virgin Islands monitored persons at low risk. Forty-four states, the District of Columbia, New York City, and Puerto Rico monitored one or more persons at some or high risk. Three territories and three freely associated states had no persons under monitoring. Approximately half (53\%) of all persons were monitored in five jurisdictions (New York City, Maryland, Georgia, Pennsylvania, and Virginia). New York City monitored the largest number of persons, followed by Maryland and Georgia (Figure 2).

\section{Discussion}

Fifty states and two local jurisdictions effectively monitored travelers arriving in the United States from Ebola-affected West African countries within 7 days of the release of updated CDC guidance on movement and monitoring on October 27, 2014; by the end of December 2014, all U.S. territories also 
FIGURE 2. Number of persons ( $N=29,789$ ) with potential exposure who were monitored for Ebola virus, by jurisdiction - United States, November 3, 2014-December 28, 2015

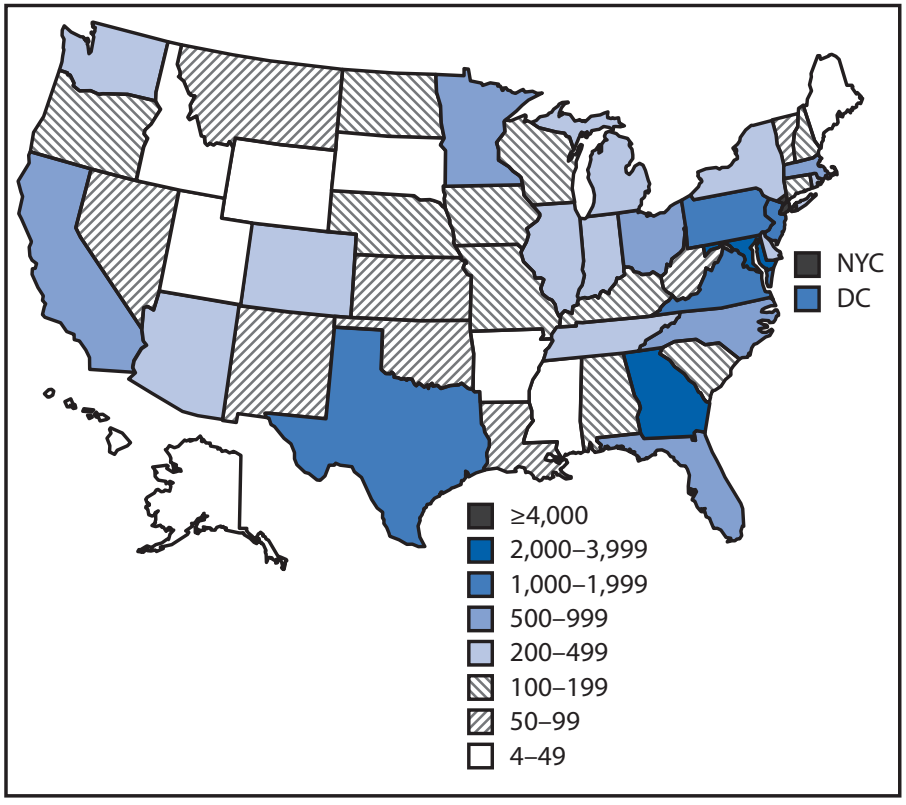

Abbreviations: DC = District of Columbia; NYC = New York City.

were reporting to CDC (2). The Movement and Monitoring Unit under the leadership of CDC's State Coordination Task Force assumed responsibility for coordinating the national response to monitor persons with potential exposure to Ebola. The Movement and Monitoring Unit 1) communicated CDC's movement and monitoring guidance to all partners, 2) activated monitoring, 3) collected and compiled reports from states and local health departments, and 4) provided information on the monitoring status of persons with risk for Ebola exposure to CDC, the U.S. Department of Health and Human Services, and the White House. As a result of this sustained effort, almost 30,000 travelers from Ebola-affected countries were monitored in the United States.

The findings in this report are subject to at least two limitations. First, weekly aggregate numbers masked precision and could be inexact. This might have occurred when a person's risk was reclassified or when individual-level daily reporting shifted to weekly reporting. Second, accounting for duplicate reporting of monitoring status was challenging. For example, aggregate weekly reporting could underestimate or overestimate monitoring numbers if a person transferred jurisdictions and was reported by both jurisdictions or by neither jurisdiction. However, efforts were made to remove duplicates from the analysis.

The overall success in monitoring $>99 \%$ of incoming travelers resulted, in part, because of the vigilance of state, local, and

\section{Summary \\ What is already known about this topic?}

Beginning in March 2014, West Africa (primarily the countries of Guinea, Liberia, and Sierra Leone) has experienced the largest outbreak of Ebola virus disease (Ebola) in history. During March 25, 2014-April 13, 2016, a total of 28,616 cases of Ebola were reported in West Africa, and 11,310 persons died. In October 2014, after the first case of imported Ebola in the United States, CDC issued monitoring and movement guidance. This guidance provided recommendations for U.S. monitoring of persons potentially exposed to Ebola.

What is added by this report?

Overall, 29,789 persons were monitored, with >99\% completing 21-day monitoring with no loss to follow-up exceeding 48 hours. In a given reporting week, a median of 1,680 persons were monitored and approximately half (53\%) of all persons were monitored in five jurisdictions. Among 796 symptomatic persons in the low-risk and some-risk categories, 104 (13\%) were tested for Ebola during their monitoring period; none tested positive for Ebola.

What are the implications for public health practice?

The overall success in monitoring $>99 \%$ of incoming travelers resulted, in part, because of the vigilance of state, local, and territorial health departments and the preparedness infrastructure that enabled jurisdictions to fully implement CDC guidance for monitoring of persons with potential Ebola exposure.

territorial health departments and the preparedness infrastructure that enabled jurisdictions to fully implement and follow CDC guidance on monitoring of persons with potential Ebola exposure. This monitoring success also can be attributed to a range of methodologies and resources used throughout the implementation period, including an enhanced entry riskassessment process that provided Check and Report Ebola kits and mobile telephones to all incoming travelers requiring monitoring, and collected personal locating information including telephone numbers, e-mail and physical addresses, and emergency contact information. Loss to follow-up was minimized by state and local health department partnerships with local police departments and Homeland Security's state fusion centers. Novel methods to contact persons via social media further facilitated communication and monitoring efforts. In most cases, initial failures in contact or loss to follow-up were attributed to missing or erroneous contact information, which can occur even with robust protocols. The monitoring of travelers from Ebola-affected countries exemplified a complex coordination of multiple agencies at multiple levels to successfully eliminate further cases of imported Ebola virus disease in the United States. 


\section{Acknowledgments}

The 60 state, local, and territorial jurisdictions that conducted monitoring during the response; Tasha Stehling-Ariza, $\mathrm{PhD}$, Division of Global Health Protection, Center for Global Health, CDC; Christine Kosmos, MS, Randolph Daley, DVM, Mary Dott, MD, Sara Vagi, PhD, CDC Office of Public Health Preparedness and Response investigators; staff members of CDC's State Coordination Task Force and Global Migration Task Force, National Center for Emerging and Zoonotic Diseases; Becky Maholland, MS, Division of Emergency Operations, Office of Public Health Preparedness and Response, CDC.
${ }^{1}$ Division of State and Local Readiness, Office of Public Health Preparedness and Response, CDC.

Corresponding author: Hyacinte Julien Kabore, yti2@cdc.gov, 404-639-3427.

\section{References}

1. Notes on the interim US guidance for monitoring and movement of persons with potential Ebola virus exposure. Atlanta, GA: US Department of Health and Human Services, CDC; 2016. http://www.cdc.gov/vhf/ebola/exposure/ monitoring-and-movement-of-persons-with-exposure.html

2. Stehling-Ariza T, Fisher E, Vagi S, et al. Monitoring of persons with risk for exposure to Ebola virus disease-United States, November 3, 2014March 8, 2015. MMWR Morb Mortal Wkly Rep 2015;64:685-9. 